\title{
GENERALIZED HOPF MANIFOLDS, LOCALLY CONFORMAL KAEHLER STRUCTURES AND REAL HYPERSURFACES
}

\author{
BY SORIN Dragomir
}

\begin{abstract}
We study the geometry of submanifolds of complex Hopf manifolds endowed with the (locally conformal Kaehler) Boothby metric.
\end{abstract}

\section{Generalized Hopf manifolds and the Boothby metric.}

Let $a \in C, 0<|a|<1$, be a fixed complex number; let $G_{a}$ be the discrete group of complex analytic transformations of $W=C^{n}-\{0\}, n>1$, generated by $z \mapsto a z, z \in W$. Then $G_{a}$ acts freely and properly discontinuously on $W$, see [28], vol. II, p. 137, so that the quotient space $\boldsymbol{H}_{a}^{n}=W / G_{a}$ becomes in a natural way a complex $n$-dimensional manifold. This is the well known complex Hopf manifold. In their attempt to construct complex structures on products $S^{1} \times L$, where $S^{1}$ is the unit circle and $L$ an odd dimensional homotopy sphere, E. Brieskorn \& A. Van de Ven, [3], have generalized Hopf manifolds a follows. Let $n \geqq 1$ and $\left(b_{0}, \cdots, b_{n}\right) \in Z^{n+1}, b_{\jmath} \geqq 1,0 \leqq \jmath \leqq n$. Let $\left(z_{0}, \cdots, z_{n}\right)$ be the natural complex coordinates on $\boldsymbol{C}^{n+1}$. Define $X^{2 n}(b)=X^{2 n}\left(b_{0}, \cdots, b_{n}\right) \subset \boldsymbol{C}^{n+1}$ by the equation :

$$
\left(z_{0}\right)^{b_{0}}+\cdots+\left(z_{n}\right)^{b_{n}}=0
$$

Then $X^{2 n}(b)$ ia an affine algebraic variety with one singular point at the origin of $\boldsymbol{C}^{n+1}$ if $b_{\jmath} \geqq 2, j=0, \cdots n$ (and without singularities if $b_{\jmath}=1$ for at least one $j)$. Next $B^{2 n}(b)=X^{2 n}(b)-\{0\}$ is a complex $n$-dimensional manifold, referred hereafter as the Brieskorn manifold determined by the integers $b_{0}, \cdots, b_{n}$. See [2]. There is a natural holomorphic action of $\boldsymbol{C}$ on $\boldsymbol{B}^{2 n}(b)$ given by:

$$
t\left(z_{0}, \cdots, z_{n}\right)=\left(z_{0} \exp \left(-\frac{t w_{a}}{b_{0}}\right), \cdots, z_{n} \exp \left(-\frac{t w_{a}}{b_{n}}\right)\right)
$$

where $t \in C, w_{a}=-\log |a|-i \Phi_{a}, \Phi_{a}=\arctan (\operatorname{Im}(a) / \operatorname{Re}(a)), \quad-\pi / 2<\Phi_{a}<\pi / 2$,

Received July 4, 1990; revised December 25, 1990. 
$i=\sqrt{-1}$. Then $\boldsymbol{Z}$ acts freely and properly discontinuously on $\boldsymbol{B}^{2 n}(b)$ as a subgroup of $\boldsymbol{C}$. The resulting complex manifold:

$$
\boldsymbol{H}_{a}^{n}(b)=B^{2 n}(b) / \boldsymbol{Z}
$$

is referred to as the generalized $\operatorname{Hopf}\left(\right.$ g.H.) manifold determined by $a$ and $b_{\jmath}$, $0 \leqq j \leqq n$. Note that $\boldsymbol{H}_{a}^{n}(1, \cdots, 1)$ and $W / G_{a}$ are diffeomorphic.

Let $D^{1}$ be the punctured open unit disk in $C$. Consider :

$$
f: D^{1} \times B^{2 n}(b) \longrightarrow D^{1} \times B^{2 n}(b)
$$

defined by $f(a, x)=\left(a, U_{a} x\right)$, for any $a \in D^{1}, x \in B^{2 n}(b)$, where $U_{a} \in G L(n+1, \boldsymbol{C})$ is the matrix :

$$
U_{a}=\operatorname{diag}\left(\exp \left(-\frac{w_{a}}{b_{0}}\right), \cdots, \exp \left(-\frac{w_{a}}{b_{n}}\right)\right)
$$

Note that $f$ is an automorphism of $D^{1} \times B^{2 n}(b)$. The action of $G L(n+1, C)$ on $\boldsymbol{C}^{n+1}$ induces an action of $\boldsymbol{Z} \approx\left\{f^{m} / m \in Z\right\}$ on $D^{1} \times B^{2 n}(b)$. Let :

$$
X^{n}=\left(D^{1} \times B^{2 n}(b)\right) / Z
$$

be the quotient space. We establish the following:

THEOREM 1. $X^{n}$ is a complex n-dimensional manifold. Moreover, if $n=2$, then there exists a surjective holomorphic map $\pi: X^{2} \rightarrow D^{1}$ which makes $X^{2}$ into a complex analytic family of compact complex surfaces, for any $a \in D^{1}$ there is a diffeomorphism between $\pi^{-1}(a)$ and $\boldsymbol{H}_{a}^{2}(b)$.

We recall that a triple $(X, \pi, M)$ is a complex analytic family of compact complex manifolds if $X, M$ are complex manifolds and $\pi: X \rightarrow M$ is a proper holomorphic map which is of maximal rank at all points of $X$. Then each fibre $\pi^{-1}(a), a \in M$, is a compact complex manifold. Note that the action (1) of $\boldsymbol{Z}$ on $B^{2 n}(b)$ generalizes slightly the one in [3], p. 390. There $B^{2 n}(1, \cdots, 1) / Z$ is diffeomorphic to $W / G_{1 / e}$. The proof of Theorem 1 is organized in several steps, as follows.

STEP 1. $\quad Z$ acts freely on $D^{1} \times B^{2 n}(b)$.

Let $(a, x)$ be a fixed point of $f^{m}, m \in Z$. Thus $U_{a}^{m} x=x$, and consequently :

$$
z_{\jmath} \exp \left(-\frac{m w_{a}}{b_{\jmath}}\right)=z_{\jmath}
$$

for $0 \leqq j \leqq n$. Since at least one $z$, is non-zero, it follows that $m=0$.

STEP 2. $\left\{f^{m} / m \in \boldsymbol{Z}\right\}$ is a properly discontinuous group of analytic transformations of $D^{1} \times B^{2 n}(b)$.

Let $K \subset D^{1}, L \subset B^{2 n}(b)$ be compact subsets. It is enough to show that the set of all $m \in \boldsymbol{Z}$ with the property: 


$$
f^{m}(K \times L) \cap(K \times L) \neq \varnothing
$$

is finite. Since $K$ is compact, there is $c>0$ such that $|a| \leqq c<1$ for any $a \in K$. Let us endow $\boldsymbol{C}^{n+1}$ with the norm:

$$
|x|=\left|z_{0}\right|+\cdots+\left|z_{n}\right|
$$

where $x=\left(z_{0}, \cdots, z_{n}\right) \in C^{n+1}$. As $L$ is compact, there are $A>0, B>0$ such that $A \leqq|x| \leqq B$ for any $x \in L$. Then:

$$
\left|U_{a}^{m} x\right|=\sum_{j=0}^{n}\left|z_{j}\right||a|^{m / b_{j}} \leqq B \sum_{j=0}^{n} c^{m / b_{\jmath}} \longrightarrow 0
$$

as $m \rightarrow+\infty$. Consequently, there is $N \in Z, N \geqq 0$, such that :

$$
\left|U_{a}^{m} x\right|<A
$$

for any $m \geqq N$. Next, we shall show that there is $N^{\prime} \in Z, N^{\prime} \geqq 0$, such that:

$$
\left|U_{a}^{-m} x\right|>B
$$

for any $(a, x) \in K \times L$ and any $m \geqq N^{\prime}$. The proof is by contradiction. Assume that there exists a sequence $\left\{m_{s}\right\}_{s \geq 1}, m_{s} \in \boldsymbol{Z}, m_{1}<m_{2}<\cdots$, and a sequence $\left\{\left(a_{s}, x_{s}\right)\right\}_{s \geq 1},\left(a_{s}, x_{s}\right) \in K \times L$, such that:

$$
\left|U_{s}^{-m_{s}} x_{s}\right| \leqq B
$$

for any $s \geqq 1$. Here $U_{s}=U_{a_{s}}$. Set $y_{s}=U_{s}^{-m_{8}} x_{s}, y_{s}=\left(\zeta_{0}^{(s)}, \cdots, \zeta_{n}^{(s)}\right)$. Hence :

$$
x_{s}=\left(\zeta_{0}^{(s)} \exp \left(-\frac{w_{s} m_{s}}{b_{0}}\right), \cdots, \zeta_{n}^{(s)} \exp \left(-\frac{w_{s} m_{s}}{b_{n}}\right)\right)
$$

where $w_{s}=w_{a_{s}}$. Thus :

$$
\left|x_{s}\right|=\sum_{j=0}^{n}\left|\zeta_{j}^{(s)}\right|\left|a_{s}\right|^{m_{s} / b} \leqq B \sum_{j=0}^{n} c^{m_{s} / b j} \longrightarrow 0
$$

as $s \rightarrow+\infty$. Thus $0 \in L \subset B^{2 n}(b)$, a contradiction. Let $m \in \boldsymbol{Z}$ such that $f^{m}(K \times L)$ $\cap(K \times L) \neq \varnothing$. Then there is $(a, x) \in K \times L$ such that $\left(a, U_{a}^{m} x\right) \in K \times L$. Assume $m \geqq N$. Then $\left|U_{a}^{m} x\right|<A$ by (2), a contradiction. Similarly, the assumption $m \leqq-N^{\prime}$ leads to a contradiction by (3). It remains that $-N^{\prime}<m<N$, i.e. the set $\left\{m \in \boldsymbol{Z} / f^{m}(K \times L) \cap(K \times L) \neq \varnothing\right\}$ is finite.

Thus $X^{n}$ is a complex manifold. Let $p: D^{1} \times B^{2 n}(b) \rightarrow X^{n}$ be the natural surjection. Moreover, let $\tilde{\pi}: D^{1} \times B^{2 n}(b) \rightarrow D^{1}, \tilde{\pi}(a, x)=a$. As $\tilde{\pi} f=\tilde{\pi}$, there exists a map $\pi: X^{n} \rightarrow D^{1}$ so that $\pi p=\tilde{\pi}$. As $p$ is a covering map, it follows that $\pi$ is surjective, holomorphic and of maximal rank (at all points of $X^{n}$ ).

Assume from now on that $n=2$, i.e. each $\pi^{-1}(a), a \in D^{1}$, is a complex surface. 
STEP 3. $\pi: X^{2} \rightarrow D^{1}$ is a proper map.

Let $K \subset D^{1}$ be a compact subset. We shall show that $\pi^{-1}(K)$ is compact in $X^{2}$. To this end, one shows that any sequence $\left\{P_{s}\right\}_{s \geq 1}$ in $\pi^{-1}(K)$ admits some convergent subsequence. Set $a_{s}=\pi\left(P_{s}\right), s \geqq 1$. Then, by passing to some subsequence if necessary, one may assume that $a_{8}$ converges to some $a \in K$, as $s \rightarrow+\infty$. Thus there are $c_{1}>0, c_{2}>0$ such that:

$$
c_{1} \leqq\left|a_{s}\right|^{1 / b_{j}} \leqq 1
$$

for any $s \geqq 1, j=0,1,2$. Choose $x_{s} \in B^{4}(b)$ such that $p\left(a_{s}, x_{s}\right)=P_{s}$; set $x_{s}=$ $\left(z_{0}^{(s)}, z_{1}^{(s)}, z_{2}^{(8)}\right)$. $1,2, \cdots$.

CASE 1. There exists a sequence $s_{1}<s_{2}<\cdots$ so that $z_{2}^{\left(s_{k}\right)}=0$, for any $k=$

As $x_{s_{k}} \in B^{4}\left(b_{0}, b_{1}, b_{2}\right)$ it follows that $\left(z_{0}^{\left(s_{k}\right)}, z_{1}^{\left(s_{k}\right)}\right) \in B^{2}\left(b_{0}, b_{1}\right)$. Thus $z_{0}^{\left(s_{k}\right)} \neq 0$, $z_{1}^{\left(s_{k}\right)} \neq 0$, for any $k=1,2, \cdots$. Note that for any $0<r<1, \rho>0$, there exists $m \in Z$ so that $r \leqq r^{m} \rho \leqq 1$. Apply this for :

$$
r=\left|a_{s_{k}}\right|^{1 / b 1}, \quad \rho=\left|z_{1}^{\left(s_{k}\right)}\right|
$$

Then there exists $m_{k} \in \boldsymbol{Z}$ so that:

$$
c_{1} \leqq\left|a_{s_{k}}\right|^{1 / b_{1}} \leqq\left|a_{s_{k}}\right|^{m_{k} / b_{1}}\left|z_{1}^{\left(s_{k}\right)}\right| \leqq 1
$$

Set :

$$
y_{s_{k}}=U_{k}^{m_{k}} x_{s_{k}}
$$

for $k=1,2, \cdots$. Here $U_{k}=U_{s_{k}}$. Then :

Set :

$$
P_{s_{k}}=p\left(a_{s_{k}}, y_{s_{k}}\right)
$$

$$
\zeta_{j}^{\left(\delta_{k}\right)}=z_{j}^{\left(8_{k}\right)} \exp \left(-\frac{m_{k} w_{\delta_{k}}}{b_{j}}\right)
$$

for $j=0,1$. By (5) one has :

$$
c_{1} \leqq\left|\zeta_{1}^{\left(s_{k}\right)}\right| \leqq 1
$$

and thus one may assume that:

$$
\zeta_{1}^{\left(s_{k}\right)} \longrightarrow z_{1}, \quad k \rightarrow+\infty
$$

for some $z_{1} \in C, c_{1} \leqq\left|z_{1}\right| \leqq 1$. Set :

As $\bar{C}$ is compact, then :

$$
\bar{C}=C \cup\{\infty\}
$$

$$
\zeta_{0}^{\left({ }^{8} k\right)} \longrightarrow z_{0}, \quad k \rightarrow+\infty
$$

for some $z_{0} \in \bar{C}$. If $z_{0} \neq \infty$, set $x=\left(z_{0}, z_{1}, 0\right)$. Then $x \in B^{4}(b)$ and $P_{s_{k}} \rightarrow p(a, x)$, as $k \rightarrow+\infty$. Note that $z_{0}=\infty$ does not occur since $\left(z_{0}\right)^{b_{0}}+\left(z_{1}\right)^{b_{1}}=0$ and $z_{1} \neq \infty$. 
CASE 2. For any $s \geqq 1, z_{2}^{(s)} \neq 0$.

Then there exists $m_{s} \in \boldsymbol{Z}$ so that:

$$
c_{1} \leqq\left|a_{s}\right|^{1 / b_{2}} \leqq\left|a_{s}\right|^{m_{s} / b_{2}}\left|z_{2}^{(s)}\right| \leqq 1
$$

for any $s \geqq 1$. By replacing $x_{s}$ with $U_{s}^{m_{s}} x_{s}$ if necessary (this is always possible due to the invariance of $p$ under the action of $Z$ on $D^{1} \times B^{4}(b)$ ) we may assume that:

Thus :

$$
c_{1} \leqq\left|z_{2}^{(s)}\right| \leqq 1, \quad s \geqq 1
$$

$$
z_{2}^{(s)} \longrightarrow z_{2}, \quad s \rightarrow+\infty
$$

for some $z_{2} \in C, c_{1} \leqq\left|z_{2}\right| \leqq 1$. As $\bar{C}$ is compact :

$$
z_{j}^{(s)} \longrightarrow z_{\jmath}, \quad s \rightarrow+\infty
$$

for some $z_{j} \in \bar{C}, j=0,1$. If $z_{0} \neq \infty$ then $z_{1} \neq \infty$, since $\left(z_{0}\right)^{b_{0}}+\left(z_{1}\right)^{b_{1}}+\left(z_{2}\right)^{b_{2}}=0$. Then set $x=\left(z_{0}, z_{1}, z_{2}\right)$. Clearly :

$$
P_{s} \longrightarrow p(a, x), \quad s \rightarrow+\infty
$$

If $z_{0}=\infty$ then $z_{1}=\infty$, too, and we may assume that:

$$
1 \leqq\left|z_{j}^{(1)}\right| \leqq\left|z_{j}^{(2)}\right| \leqq \cdots \rightarrow+\infty
$$

for $j=0,1$. There exists $m_{s} \in \boldsymbol{Z}$ such that:

for all $s \geqq 1$. Set :

$$
c_{1} \leqq\left|a_{s}\right|^{1 / b_{1}} \leqq\left|a_{s}\right|^{m_{s} / b_{1}}\left|z_{1}^{(s)}\right| \leqq 1
$$

$$
\xi_{\jmath}^{(s)}=z_{\jmath}^{(s)} \exp \left(-\frac{m_{s} w_{s}}{b_{\jmath}}\right)
$$

for $j=0,1,2$. By $(7)$ one has $c_{1} \leqq\left|\xi_{1}^{(s)}\right| \leqq 1$. Also $c_{1} \leqq\left|z_{2}^{(s)}\right| \leqq 1$ yields $0 \leqq\left|\xi_{2}^{(s)}\right|$ $\leqq 1$. As $\left|\xi_{0}^{(s)}\right|>0$ there is $m_{s}^{\prime} \in \boldsymbol{Z}$ such that:

Set :

$$
c_{1} \leqq\left|a_{s}\right|^{1 / b_{0}} \leqq\left|a_{s}\right|^{m_{s}^{\prime} / b_{0}}\left|\xi_{0}^{(s)}\right| \leqq 1
$$

$$
u_{j}^{(s)}=\xi_{j}^{(s)} \exp \left(-\frac{m_{s}^{\prime} w_{s}}{b_{\jmath}}\right)
$$

for $j=0,1,2$. By (8) one has :

Also :

$$
c_{1} \leqq\left|u_{0}^{(s)}\right| \leqq 1
$$

$$
0<\left|u_{j}^{(s)}\right| \leqq 1
$$

for $j=1,2$. Therefore, one may assume that $u_{0}^{(s)} \rightarrow u_{0}$ and $u_{j}^{(s)} \rightarrow u$, for some $u_{0}, u_{j} \in C, c_{1} \leqq\left|u_{0}\right| \leqq 1,0 \leqq\left|u_{j}\right| \leqq 1, j=1,2$. Set $x^{\prime}=\left(u_{0}, u_{1}, u_{2}\right)$. Then $x^{\prime} \in B^{4}(b)$ and $P_{s} \rightarrow p\left(a, x^{\prime}\right)$, as $s \rightarrow+\infty$. 
Thus $\left(X^{2}, \pi, D^{1}\right)$ is a complex analytic family of compact complex surfaces. Actually, each fibre $\pi^{-1}(a)$ may be identified with the g. H. surface $\boldsymbol{H}_{a}^{2}\left(b_{0}, b_{1}\right)$ by $p(a, x) \mapsto p_{a}(x)$, where $p_{a}: B^{4}(b) \rightarrow \boldsymbol{H}_{a}^{2}(b)$ is the natural covering map. Our Theorem 1 is completely proved.

Let $\left(M^{2 n}, g_{0}, J\right)$ be a Hermitian manifold of complex dimension $n \geqq 2$, where $g_{0}$ denotes the Hermitian metric, while $J$ stands for the complex structure. Let $\Omega_{0}$ be its Kaehler 2-form, i.e. $\Omega_{0}(X, Y)=g_{0}(X, J Y)$. Then $M^{2 n}$ is a locally conformal Kaehler (l.c.K.) manifold if :

$$
d \Omega_{0}=\omega_{0} \wedge \Omega_{0}
$$

for some closed globally defined 1-form $\omega_{0}$ on $M^{2 n}$. See e.g. T. Kashiwada, [27].

Let $\left(M^{2 n}, g_{0}, J\right)$ be a Hermitian manifold. If a globally defined 1-form $\omega_{0}$ satisfying (9) exists, then it is uniquely determined, i.e.

$$
\omega_{0}=\frac{1}{n-1}\left(\delta \Omega_{0}\right) J
$$

where $\delta$ denotes the usual codifferentiation operator (the adjoint of $d$ with respect to $g_{0}$ ). For instance, if $M^{4}$ is a Hermitian surface (i.e. $n=2$ ) then $\omega_{0}$ defined by (10) satisfies (9), yet generally $\omega_{0}$ is not closed. See [53].

Let $M^{2 n}$ be a 1.c. K. manifold. The 1 -form (10) is the Lee form of $M^{2 n}$. Clearly, if $\omega_{0}=0$ then $g_{0}$ falls into nothing but a Kaehler metric. Several examples of 1.c.K. manifolds which admit no global Kaehler metrics are known, see e.g. F. Tricerri, [47].

Let $M^{2 n}$ be a l.c.K. manifold and let $\bar{\nabla}$ be the Riemannian connection of $g_{0}$. We shall need the Weyl connection $\vec{D}$, i. e. the torsion-free linear connection on $M^{2 n}$ expressed by :

$$
\bar{D}_{X} Y=\bar{\nabla}_{X} Y-\frac{1}{2}\left\{\omega_{0}(X) Y+\omega_{0}(Y) X-g_{0}(X, Y) B_{0}\right\}
$$

Here $B_{0}=\left(\omega_{0}\right) \#$ is the Lee field. Also \# denotes raising of indices with respect to $g_{0}$, i. e. $g_{0}\left(X, \lambda^{\#}\right)=\lambda(X)$, where $\lambda$ is any 1 -form on $M^{2 n}$. Let $\bar{K}, \bar{R}$ be respectively the curvature tensor fields of $\bar{D}$ and $\bar{\nabla}$. As a consequence of (11) one has :

$$
\begin{aligned}
\bar{K}(X, Y) Z= & \bar{R}(X, Y) Z-\frac{1}{2}\left\{L_{0}(X, Z) Y\right. \\
& -L_{0}(Y, Z) X+g_{0}(X, Z) L_{0}(Y, \cdot)^{\#} \\
& \left.-g_{0}(Y, Z) L_{0}(X, \cdot)^{\#}\right\}-\frac{c^{2}}{4}(X \wedge Y) Z
\end{aligned}
$$

where :

$$
L_{0}=\bar{\nabla} \omega_{0}+\frac{1}{2} \omega_{0} \otimes \omega_{0}
$$


and $(X \wedge Y) Z=g_{0}(Y, Z) X-g_{0}(X, Z) Y$; also $c=\left\|\omega_{0}\right\|, c \in C^{\infty}\left(M^{2 n}\right)$.

A PK-manifold (cf. the terminology in [49]) is a 1.c.K. manifold whose Lee form is parallel (with respect to $\bar{\nabla}$ ). If $M^{2 n}$ is a $P K$-manifold then $c \in \boldsymbol{R}$.

Let $M^{2 n}$ be a 1.c. K. manifold. As $d \omega_{0}=0$, by the classical Poincare lemma, there exists an open covering $\left\{U_{i}\right\}_{i \in I}$ of $M^{2 n}$ and a family $\left\{f_{\imath}\right\}_{i \in I}$ of smooth real valued functions, $f_{i} \in C^{\infty}\left(U_{\imath}\right), i \in I$, such that:

$$
\left(\omega_{0}\right)_{x}=\left(d f_{i}\right)_{x}
$$

for any $x \in U_{\imath}$. Let us consider the local metrics $g_{i}=\exp \left(-f_{\imath}\right) g_{0}, i \in I$. Each $\left(U_{i}, g_{i}, J\right)$ is a Hermitian manifold. Let $\Omega_{\imath}$ be its Kaehler 2-form. Then (9) yields $d \Omega_{i}=0$, i.e. $g_{i}$ is a (local) Kaehler metric. Any such two metrics are conformally related, i.e. $g_{\jmath}=\exp \left(f_{i}-f_{\jmath}\right) g_{i}$ on $U_{i} \cap U_{\jmath}$, and thus (being Kaehler) they are homothetic, i.e.

$$
f_{i}-f_{j}=c_{\imath j}, \quad c_{\imath j} \in \boldsymbol{R}
$$

This agrees with (14). Let $\nabla^{i}$ be the Riemannian connection of $\left(U_{i}, g_{i}\right)$. Then :

$$
\nabla_{X}^{i} Y=\bar{\nabla}_{X} Y-\frac{1}{2}\left\{X\left(f_{i}\right) Y+Y\left(f_{\imath}\right) X-g_{0}(X, Y) \operatorname{grad}\left(f_{\imath}\right)\right\}
$$

where $\operatorname{grad}\left(f_{i}\right)=\left(d f_{i}\right)^{\#}$. Consequently, the local connections $\nabla^{i}$ glue up to $\bar{D}$, i. e. the Weyl connection is precisely the Riemannian connection of the local metrics $g_{i}, i \in I$. Since these are Kaehler, it follows :

$$
\bar{D} J=0
$$

Let $M^{2 n}$ be a $P K$-manifold. It is said to be a $P_{0} K$-manifold (cf. [49]) if its local Kaehler metrics $\left\{g_{i}\right\}_{i \in I}$ are flat, i. e. $\bar{K}=0$. Thus, by (12), the curvature $\bar{R}$ of a $P_{0} K$-manifold has the following expression:

$$
\begin{aligned}
\bar{R}(X, Y) Z= & \frac{c^{2}}{4}(X \wedge Y) Z \\
& +\frac{1}{4}\left\{\left[\omega_{0}(X) Y-\omega_{0}(Y) X\right] \omega_{0}(Z)\right. \\
& \left.+\left[g_{0}(X, Z) \omega_{0}(Y)-g_{0}(Y, Z) \omega_{0}(X)\right] B_{0}\right\}
\end{aligned}
$$

Our standard example of $P_{0} K$-manifold is the complex Hopf manifold $\boldsymbol{H}_{a}^{n} \approx$ $\boldsymbol{H}_{a}^{n}(1, \cdots, 1)$; let us consider the Hermitian metric :

$$
d s^{2}=|z|^{-2} \delta_{i j} d z^{2} \otimes d z^{\jmath}
$$

on $W=C^{n}-\{0\}$, where $|z|^{2}=\delta_{i j} z^{2} \bar{z}^{j}, z=\left(z^{1}, \cdots, z^{n}\right), z \in W$. Note that (17) is $G_{a}$-invariant and thus determines a (globally defined) Hermitian metric $g_{0}$ on $\boldsymbol{H}_{a}^{n}$. This was discovered by W. Boothby, [1], for $n=2$. It was observed by I. Vaisman, [49], [52], that actually $g_{0}$ is a l.c. K. metric on $\boldsymbol{H}_{a}^{n}$. It is referred to as the Boothby metric of $\boldsymbol{H}_{a}^{n}$. It is known that $\boldsymbol{H}_{a}^{n} \approx S^{1} \times S^{2 n-1}$ (a diffeomor- 
phism); thus $\boldsymbol{H}_{a}^{n}$ is compact. It follows also that the first Betti number is $b_{1}\left(\boldsymbol{H}_{a}^{n}\right)=1$, so that $\boldsymbol{H}_{a}^{n}$ admits no global Kaehler metrics. It is easily verified that the Lee form (locally given by $\left.\omega_{0}=d \log |z|^{2}\right)$ of $\left(\boldsymbol{H}_{a}^{n}, g_{0}\right)$ is parallel.

Let $C I^{2}$ be the Inoue complex surface, cf. M. Inoue, [25]; let $(z, w)$ be complex analytic local coordinates on $\boldsymbol{C} I^{2}$. Set $z=x+i y$. The Hermitian metric :

$$
g_{0}=y^{-2} d z \otimes d \bar{z}+y d w \otimes d \bar{w}
$$

makes $C I^{2}$ into a 1.c.K. manifold (with the Lee form $\omega_{0}=y^{-1} d y$ ). The complex Inoue surface is compact. Also $b_{1}\left(C I^{2}\right)=1$, so that $C I^{2}$ carries no globally defined Kaehler metrics. According to [47], $C I^{2}$ with the metric (18) is not a $P K$-manifold.

\section{The complex sphere.}

Let $Q_{n-1} \subset \boldsymbol{H}_{a}^{n}$ be defined by :

$$
\left(z^{1}\right)^{2}+\cdots+\left(z^{n}\right)^{2}=0
$$

Note that (19) is $G_{a}$-invariant, so that $Q_{n-1}$ is well defined. It is a complex hypersurface of $\boldsymbol{H}_{a}^{n}$ and it is referred as the complex sphere in $\boldsymbol{H}_{a}^{n}$. Let $b_{j} \in \boldsymbol{Z}$, $b_{j} \geqq 0,1 \leqq j \leqq n$. Let $B^{2 n-2}\left(b_{1}, \cdots, b_{n}\right) \subset W$ be the Brieskorn monifold determined by the integers $b_{j}$, cf. our section 1 . Let:

$$
L^{2 n-3}\left(b_{1}, \cdots, b_{n}\right)=B^{2 n-2}\left(b_{1}, \cdots, b_{n}\right) \cap S^{2 n-1}
$$

be the Brieskorn (exotic) sphere determined by $b_{3}, 1 \leqq j \leqq n$. We establish the following :

THEOREM 2. Let $a \in \boldsymbol{R}, 0<a<1$, and $n \geqq 5$. Then the complex sphere $Q_{n-1}$ in $\boldsymbol{H}_{a}^{n}$ is a compact l.c.K. manifold which admits no globally defined Kaehler metrics.

Proof. Let $\pi: W \rightarrow \boldsymbol{H}_{a}^{n}$ be the natural surjection. Define $f: \boldsymbol{H}_{a}^{n} \rightarrow S^{1} \times S^{2 n-1}$ by :

$$
f: \pi(z) \longmapsto\left(\exp \left(\frac{2 \pi i \log |z|}{\log a}\right), \frac{z}{|z|}\right)
$$

for any $z \in W$. Note that the right hand member of $(20)$ is $G_{a}$-invariant, i.e. $f(\pi(z))$ is well defined. Then $f$ is a diffeomorphism with the obvious inverse:

$$
f^{-1}:(w, \zeta) \longmapsto \pi\left(a^{\arg (w) / 2 \pi} \zeta\right)
$$

for any $w \in S^{1}, \zeta \in S^{2 n-1}$. Then (20) induces a diffeomorphism:

$$
Q_{n-1} \approx S^{1} \times L^{2 n-3}(2, \cdots, 2)
$$


By Corollary 2.10 in [11], p. 58, it follows :

$$
H_{j}\left(Q_{n-1} ; \boldsymbol{Z}\right) \approx H_{\jmath-1}\left(L^{2 n-3} ; \boldsymbol{Z}\right) \oplus H_{j}\left(L^{2 n-3} ; \boldsymbol{Z}\right)
$$

where $L^{2 n-3}$ is short for $L^{2 n-3}(2, \cdots, 2)$. Let $G$ be any abelian group; by the universal coefficient theorem (e.g. [35], p. 332) the sequence :

$$
0 \longrightarrow H_{2}\left(Q_{n-1} ; Z\right) \otimes G \longrightarrow H_{2}\left(Q_{n-1} ; G\right) \longrightarrow \operatorname{Tor}\left(H_{1}\left(Q_{n-1} ; Z\right), G\right) \longrightarrow 0
$$

is exact. Set $j=2$ in (23); by a result of E. Brieskorn, [2], one has:

$$
H_{i}\left(L^{2 n-3} ; \boldsymbol{Z}\right)=0, \quad i=1,2
$$

provided that $n \geqq 5$. Thus $H_{2}\left(Q_{n-1} ; \boldsymbol{Z}\right)=0$. Set $\jmath=1$ in $(21)$; as $L^{2 n-3}$ is connected and (24) exact, it follows :

$$
H_{2}\left(Q_{n-1} ; G\right) \approx \operatorname{Tor}(\boldsymbol{Z}, G)=0
$$

Assume further that $G$ is a principal ideal domain (e.g. $\boldsymbol{Z}$ or a field). Then $H^{2}\left(Q_{n-1} ; G\right) \approx \operatorname{Hom}\left(H_{2}\left(Q_{n-1} ; G\right), G\right)=0$, (cf. e.g. [34], p. 259). In particular $H^{2}\left(Q_{n-1} ; \boldsymbol{R}\right)=0$. Therefore $Q_{n-1}$ is a compact (by (22)) complex manifold which carries no globally defined Kaehler metric. Clearly $Q_{n-1}$ inherits a l.c.K. structure as a complex submanifold of $\boldsymbol{H}_{a}^{n}$.

Remarks. 1) Note that the complex sphere $Q_{n-1}$ is a g. H. manifold $H_{a}^{n-1}(2, \cdots, 2)$, cf. our section 1. E. Brieskorn \& A. Van de Ven, [3], show that each product between $S^{1}$ and a $(2 n-1)$-homotopy sphere, $n \neq 2$, bounding a parallelizable manifold carries a complex structure in a natural way. If $b_{j}=2$, $1 \leqq j \leqq n$, our Theorem 2 completes this result, i.e. puts a Riemannian metric on $H_{a}^{n-1}(2, \cdots, 2)$ which is 1 .c. K. with respect to the complex structure discovered in [3].

2) One may show that $b_{2}\left(L^{2 n-3}\right)=0$ by using results in differential geometry as follows. By a result of Y. Tashiro, [46], $L^{2 n-3}$ inherits an almost contact metric (a.ct.m.) structure, as a real hypersurface of the Kaehler manifold $B^{2 n-2}(2, \cdots, 2)$. Next, cf. S. Sasaki \& C. J. Hsu, [45], this a.ct. m. structure is actually Sasakian. Finally, one may use Proposition 1 of S. Goldberg, [21], p. 106 , to conclude that $b_{2}\left(L^{2 n-3}\right)=0$.

\section{Submersions from complex Hopf manifolds.}

Let $p: S^{2 n-1} \rightarrow \boldsymbol{C} P^{n-1}$ be the Hopf fibration. This is a principal $S^{1}$-bundle and a Riemannian submersion, as well. Both $S^{2 n-1}$ and $C P^{n-1}$ are thought of as being endowed with their canonical Riemannian structures, i.e. the Riemannian metric induced on $S^{2 n-1}$ by the flat Kaehler metric of $\boldsymbol{C}^{n}$, and the Fubini-Study metric, respectively. Set:

$$
D=\{2 \pi i m+(\log a) k / m, k \in \boldsymbol{Z}\}
$$


This is a well defined lattice in $C$. Consider the complex 1-torus $T_{C}^{1}=C / D$. Define $q: \boldsymbol{H}_{a}^{n} \rightarrow \boldsymbol{C} P^{n-1}$ by $q(\pi(z))=[z]$, where $[z]$ denotes the point of $\boldsymbol{C} P^{n-1}$ of homogeneous coordinates $z$. Then $q: \boldsymbol{H}_{a}^{n} \rightarrow \boldsymbol{C} P^{n-1}$ is a principal $T_{\boldsymbol{c}}^{1}$-bundle. Thus $q$ is a submersion. (Recall that for any principal $G$-bundle $P$ over some base manifold $M$, the projection $P \rightarrow M$ is a submersion; also $j: G \rightarrow P, j(g)=p_{0} g$, $g \in G$, is an immersion. Here $p_{0} \in P$ is fixed.) The action of $T_{c}^{1}$ on $\boldsymbol{H}_{a}^{n}$ is described as follows:

$$
\begin{gathered}
\boldsymbol{H}_{a}^{n} \times T_{c}^{1} \longrightarrow \boldsymbol{H}_{a}^{n} \\
(\pi(z), w+D) \longmapsto \pi(z \exp (w))
\end{gathered}
$$

Thus we have an immersion $\jmath: T_{\boldsymbol{c}}^{1} \rightarrow \boldsymbol{H}_{a}^{n}, \quad j(a+D)=\pi\left(z_{0} \exp (w)\right)$, where $z_{0} \in W$ is fixed. We obtain the following:

THEOREM 3. Let $\boldsymbol{H}_{a}^{n}, \boldsymbol{C} P^{n-1}$ be endowed with the Boothby metric and the Fubini-Study metric, respectively. Then $q: \boldsymbol{H}_{a}^{n} \rightarrow \boldsymbol{C} P^{n-1}$ is a Riemannian submersion. Moreover, its fibres are minumal (and therefore $q$ is harmonic).

Proof. Let $i: S^{2 n-1} \rightarrow C^{n}$ be the canonical inclusion; let $z_{0} \in S^{2 n-1}$ be fixed. Note that the canonical Riemannian structure of $S^{2 n-1}$ and the Riemannian structure induced by (17) actually coincide. Set:

$$
V_{z_{0}}=\operatorname{Ker}\left(d_{z_{0}} p\right)
$$

Let moreover $H_{z_{0}}$ be the orthogonal complement of $V_{z_{0}}$ in $T_{z_{0}}\left(S^{2 n-1}\right)$. The fact that $p$ is a Riemannian submersion is expressed by claiming that $d_{z_{0}} p: H_{z_{0}} \rightarrow$ $T_{p\left(z_{0}\right)}\left(\boldsymbol{C} P^{n-1}\right)$ is a linear isometry. Set:

$$
V_{0, \pi\left(z_{0}\right)}=\operatorname{Ker}\left(d_{\pi\left(z_{0}\right)} q\right)
$$

Let also $N_{z_{0}}\left(S^{2 n-1}\right)$ be the normal space on $S^{2 n-1}$ at $z_{0}$, i. e. the orthogonal complement of $(d i) T_{z_{0}}\left(S^{2 n-1}\right)$ in $T_{z_{0}}\left(\boldsymbol{C}^{n}\right)$.

LEMma 1. Let $z_{0} \in S^{2 n-1}$. Then:

$$
V_{0, \pi\left(z_{0}\right)}=\left(d_{z_{0}} \pi\right)\left[\left(\left(d_{z_{0}} i\right) V_{z_{0}}\right) \oplus N_{z_{0}}\left(S^{2 n-1}\right)\right] .
$$

Proof. Let $N$ be a unit normal field on $S^{2 n-1}$, i.e. the position vector at any point on $S^{2 n-1}$. Set $U=-J N$, where $J$ stands for the complex structure on $\boldsymbol{C}^{n}$. Then $U_{z_{0}}$ spans $V_{z_{0}}$. At this point $q \circ \pi \circ i=p$ and the fact that $U$ is vertical, yield:

$$
(d \pi)(d i) V_{z_{0}} \subset V_{0, \pi\left(z_{0}\right)}
$$

Let $\bar{p}: W \rightarrow C P^{n-1}$ be the natural map. Note that $\bar{p}$ is holomorphic. Then:

$$
\begin{aligned}
& \left(d_{\pi\left(z_{0}\right)} q\right)\left(d_{z_{0}}, \pi\right) N_{z_{0}}=\left(d_{\pi\left(z_{0}\right)} \bar{p}\right) J_{z_{0}}\left(d_{z_{0}}, i\right) U_{z_{0}} \\
& \quad=J^{\prime} \bar{p}\left(z_{0}\right) \\
& \left.\left(d_{z_{0}} \bar{p}\right)\left(d_{z_{0}} i\right) U_{z_{0}}=J_{p\left(z_{0}\right)}^{\prime}\right)\left(d_{z_{0}} p\right) U_{z_{0}}=0
\end{aligned}
$$


where $J^{\prime}$ denotes the complex structure on $C P^{n-1}$. The proof of Lemma 1 is complete.

Let $(\cdot, \cdot)_{z_{0}}$ be the inner product on $T_{z_{0}}\left(\boldsymbol{C}^{n}\right)$ induced by the canonical flat Kaehler metric of $\boldsymbol{C}^{n}$; since $\left|z_{0}\right|=1$, this coincides with the inner product induced by (17). Moreover $(d \pi)(d i): T_{z_{0}}\left(S^{2 n-1}\right) \rightarrow T_{\pi\left(z_{0}\right)}\left(\boldsymbol{H}_{a}^{n}\right)$ follows to be a linear isometry. This fact and Lemma 1 yield:

$$
H_{0, \pi\left(z_{0}\right)}=(d \pi)(d i) H_{z_{0}}
$$

where $H_{0, \pi\left(z_{0}\right)}$ denotes the orthogonal complement of $V_{0, \pi\left(z_{0}\right)}$ in $T_{\pi\left(z_{0}\right)}\left(\boldsymbol{H}_{a}^{n}\right)$. As $p$ is a Riemannian submersion, the formula (25) shows that:

$$
d_{\pi(z)} q: H_{0, \pi(z)} \longrightarrow T_{q(\pi(z))}\left(\boldsymbol{C} P^{n-1}\right)
$$

is a linear isometry, for any $z \in S^{2 n-1}$. The same holds for arbitrary $z \in W$. In order to verify this, one should firstly establish:

LEMMA 2. $T_{c}^{1} \subset \operatorname{Isom}\left(\boldsymbol{H}_{a}^{n}\right)$.

Proof. One needs to show that for each $g \in T_{c}^{1}$ the right translation $R_{\boldsymbol{g}}: \boldsymbol{H}_{a}^{n} \rightarrow \boldsymbol{H}_{a}^{n}$ is an isometry. Set $g=w+D, w \in \boldsymbol{C}$; locally $\boldsymbol{R}_{\mathrm{g}}$ is the map $z \mapsto$ $z \exp (w)$. Set $\exp (w)=u+i v$ and $z^{j}=x^{j}+i y^{j}$. THe (real) Jacobian of $R_{\boldsymbol{g}}$ is given by:

Consequently :

$$
\left(\begin{array}{rr}
u \delta_{j}^{i} & -v \delta_{j}^{i} \\
v \delta_{j}^{i} & u \delta_{j}^{i}
\end{array}\right) .
$$

$$
\begin{aligned}
& |z \exp (w)|^{-2}\left(\left(d R_{g}\right) \partial_{i},\left(d R_{g}\right) \partial_{\jmath}\right)_{z} \exp (w) \\
& \quad=|z|^{-2}\left(u^{2}+v^{2}\right)^{-1}\left(u \partial_{i}+v \partial_{\jmath}, u \partial_{j}+v \partial_{j}\right)_{s} \\
& \quad=|z|^{-2} \delta_{i j}
\end{aligned}
$$

etc. Thus $\left(d R_{g}\right): T_{\pi(z)}\left(\boldsymbol{H}_{a}^{n}\right) \rightarrow T_{\pi(z \exp (w))}\left(\boldsymbol{H}_{a}^{n}\right)$ is a linear isometry. Here $\partial_{i}=$ $\partial / \partial x^{2}, \partial_{i}=\partial / \partial y^{i}$.

As $q \circ R_{g}=$ const., each right translation preserves vertical spaces. By Lemma 2, right translations will preserve horizontal spaces, too. Then, the only fact that remains to be proved is that given arbitrarily $z \in W$, there exist $g \in T_{C}^{1}$ and $z_{0} \in S^{2 n-1}$ such that $\pi(z)=\pi\left(z_{0}\right) g$. Indeed, one may take $g=\log |z|$ $+D$, and $z_{0}=z /|z|$.

Since $w \mapsto z \exp (w), w \in C$, is holomorphic (here $z \in W$ is fixed) it follows that $T_{c}^{1}$ is a complex submanifold of $\boldsymbol{H}_{a}^{n}$.

LEMMA 3.. $T_{c}^{1}$ is tangent to the Lee field of $\boldsymbol{H}_{a}^{n}$.

Proof. Let $\left(x^{1}, \cdots, x^{2 n}\right)$ be a local system of (real analytic) coordinates on $\boldsymbol{H}_{a}^{n}$. Then the Boothby metric is locally given by $g_{i j}=|x|^{-2} \delta_{i j}$ and the Lee 
form by $\omega_{i}=2|x|^{-2} x_{i}$, where $x_{i}=x^{i}$. Consequently, the Lee field is locally given by $B^{i}=g^{i j} \omega_{j}=2 x^{i}$; set $w=x+i y$. Then :

$$
B_{j(w+D)}=(d j) \frac{\partial}{\partial x}
$$

i.e. $T_{c}^{1}$ is tangent to $B$.

Finally, one may use Theorem 1 in [13], p. 181, to conclude that $j$ is minimal. Our Theorem 3 is thereby proved.

Taking the Riemannian submersion $q: \boldsymbol{H}_{a}^{n} \rightarrow \boldsymbol{C} P^{n-1}$ as a fundamental example, it is tempting to consider more generally submersions from complex Hopf manifolds (with the Boothby metric) with an arbitrary base manifold. The classification problem for such submersions is open. See also R.H. Escobales, [16], [17].

\section{Submanifolds of locally conformal Kaehler manifolds.}

It is the proper point to establish the basic formulae for an isometric immersion in a $P_{0} K$-manifold. Let $\Psi: M^{m} \rightarrow M^{2 n}$ be an isometric immersion of a $m$-dimensional Riemannian manifold $\left(M^{m}, g\right)$ into the 1. c. K. manifold $\left(M^{2 n}, g_{0}, J\right)$. We shall need the Gauss and Weingarten formulae:

$$
\begin{gathered}
\bar{\nabla}_{X} Y=\nabla_{X} Y+h(X, Y) \\
\bar{\nabla}_{X} \xi=-A_{\xi} X+\nabla_{\bar{X}} \xi
\end{gathered}
$$

where $\nabla, h, A_{\xi}$ and $\nabla^{\perp}$ denote respectively the Riemannian connection of $g$, the second fundamental form (of $\Psi$ ), the Weingarten operator (associated with the normal section $\xi$ ) and the normal connection.

Let $M^{2 n}$ be a $P_{0} K$-manifold. By a result of I. Vaisman, [49], the universal covering space of $M^{2 n}$ is $W$ with the metric $c^{-2}|z|^{-2} \delta_{j k} d z^{3} \otimes \bar{z}^{k}$, i.e. $M^{2 n}$ is locally analitically homothetic to a complex Hopf manifold (with the Boothby metric). One adopts the notation $M^{2 n}=\boldsymbol{H}_{a, c}^{n}$. If $g_{0}$ is the Boothby metric, then $c=2$ (see e.g. [49]). Then $\boldsymbol{H}_{a, 2}^{n}=\boldsymbol{H}_{a}^{n}$ (locally).

Let $\Psi: M^{m} \rightarrow \boldsymbol{H}_{a, c}^{n}$ be an isometric immersion. As a consequence of (16), the Gauss-Ricci-Codazzi equations of $\Psi$ are :

$$
\begin{aligned}
& R(X, Y) Z= \frac{c^{2}}{4}(X \wedge Y) Z+A_{h(Y, Z)} X-A_{h(X, Z)} Y \\
&+\frac{1}{4}\{[\omega(X) Y-\omega(Y) X] \omega(Z) \\
&+[g(X, Z) \omega(Y)-g(Y, Z) \omega(X) B\} \\
&\left(\nabla_{X} h\right)(Y, Z)-\left(\nabla_{Y} h\right)(X, Z)=\frac{1}{4}[g(X, Z) \omega(Y)-g(Y, Z) \omega(X)] B^{\perp}
\end{aligned}
$$




$$
g_{0}\left(R^{\perp}(X, Y) \xi, \eta\right)=g\left(\left[A_{\xi}, A_{\eta}\right] X, Y\right)
$$

Here $R, R^{\perp}$ denote the curvature tensor fields of $\nabla, \nabla^{\perp}$. Moreover $\omega=\Psi^{*} \omega_{0}$. Next, if $E(\Psi) \rightarrow M^{m}$ is the normal bundle (of the given immersion $\Psi$ ), let $\tan _{x}$, nor $x$ be the projections associated with:

$$
T_{\Psi(x)}\left(\boldsymbol{H}_{a, c}^{n}\right)=(d \Psi) T_{x}\left(M^{m}\right) \oplus E(\Psi)_{x}
$$

for any $x \in M^{m}$. Set $B=\tan \left(B_{0}\right), B^{\perp}=\operatorname{nor}\left(B_{0}\right)$. As $\omega_{0}$ is parallel, by (26) it follows :

$$
\left(\nabla_{X} \omega\right) Y=\omega_{0}(h(X, Y)) .
$$

Note that although $\boldsymbol{H}_{a, c}^{n}, \quad c \neq 0$, is strongly non-Kaehler (i.e. $\left(\omega_{0}\right)_{x} \neq 0$, for any $\left.x \in \boldsymbol{H}_{a, c}^{n}\right)$ the induced 1-form $\boldsymbol{\omega}$ may have singular points.

Examples. 1) Let $\Psi: S^{2 n-1} \rightarrow \boldsymbol{H}_{a}^{n}$ be defined by:

$$
\Psi(\zeta)=\pi\left(\zeta a^{1 / 4}\right)
$$

for any $\zeta \in S^{2 n-1}$. Here $0<a<1$. By (21), $\Psi=f^{-1} \circ j$, where $j: S^{2 n-1} \rightarrow S^{1} \times S^{2 n-1}$, $\zeta \mapsto(i, \zeta), i=\sqrt{-1}$. Then $\Psi$ is an isometric immersion from $S^{2 n-1}$ with the canonical Riemannian structure, to $\boldsymbol{H}_{a}^{n}$ (with the Boothby metric).

2 ) Let $E^{2 n-3}=\Psi\left(S^{2 n-1}\right) \cap Q_{n-1}$. Then $E^{2 n-3}$ is a real hypersurface of the complex sphere. As totally-umbilicity is a conformal invariant, $E^{2 n-3}$ is totallyumbilical in $Q_{n-1}$.

3) Let $\boldsymbol{C}^{+}=\{z \in \boldsymbol{C} / \operatorname{Im}(z)>0\}$ be the upper half of the complex plane. As the complex Inoue surface $\boldsymbol{C} I^{2}$ is a quotient of $\boldsymbol{C}^{+} \times \boldsymbol{C}$ by some discrete group of analytic transformations (see e.g. [47]), let $\pi: \boldsymbol{C}^{+} \times \boldsymbol{C} \rightarrow \boldsymbol{C} I^{2}$ be the natural surjection (a local diffeomorphism). Let $L=\left\{z \in C^{+} / \operatorname{Im}(z)=1\right\}$ and $\Psi: L \times C$ $\rightarrow \boldsymbol{C} I^{2}, \Psi=\pi \circ j$, where $j: L \times \boldsymbol{C} \rightarrow \boldsymbol{C}^{+} \times \boldsymbol{C}$ is the natural inclusion. Then $\Psi$ is a minimal isometric immersion. Also $L \times C$ is an example of a real hypersurface of $C I^{2}$ having exactly two constant principal curvatures $\lambda=-1$ and $\mu=1 / 2$.

The study of the geometry of (the second fundamental form of) submani folds in 1.c. K. manifolds is of recent interest and has been initiated by $\mathrm{K}$. Matsumoto, [30], [31] and [33], I. Vaisman, [50], and continued further by B. Y. Chen \& P. Piccinni, [9], J. L. Cabrerizo \& M.F. Andres, [10], L. Ornea, [41], S. Ianus \& all, [24], and author [12], [13] and [14]. We may formulate the following.

THEOREM4. Let $M^{2 n-1}$ be an orientable connected real hypersurface of the $P_{0} K$-manifold $\boldsymbol{H}_{a, c}^{n}, n \geq 3$, having exactly two constant principal curvatures, both of multiplicity $\geqq 2$. Then $M^{2 n-1}$ is locally isometric to a product $L \times L^{\prime}$ of totally umbilical submanifolds of $\boldsymbol{H}_{a, c}^{n}$ having a flat normal connection. Moreover, either $M^{2 n-1}$ is tangent to the Lee field of $\boldsymbol{H}_{a, c}^{n}$ or $L, L^{\prime}$ are extrinsic spheres of $\boldsymbol{H}_{a, c}^{n}$.

THEOREM 5. Let $M^{2 n-1}$ be an orientable connected quasi-Einstein (i.e. Ric= 
$\rho g-(2 n-3 / 4) \omega \otimes \omega)$ real hypersurface of $\boldsymbol{H}_{a, c}^{n}, n \geqq 3$, tangent to the Lee field of $\boldsymbol{H}_{a, c}^{n}$.

i) If $\rho>(2 n-3) c^{2} / 4$ then $M^{2 n-1}$ is totally umbilical in $\boldsymbol{H}_{a, c}^{n}$.

ii) If $\rho=(2 n-4) c^{2} / 4$ then either $M^{2 n-1}$ is totally geodesic in $\boldsymbol{H}_{a, c}^{n}$ or it has exactly two principal curvatures, one non-zero of multiplicity 1 , and the other zero (of multiplicity $2 n-2$ ).

iii) If $\rho<(2 n-3) c^{2} / 4$ then $M^{2 n-1}$ is locally isometric to a product of two extrinsic spheres of $\boldsymbol{H}_{a, c}^{n}$.

Let $M^{2 n-1}$ be an orientable real hypersurface of $\boldsymbol{H}_{a, c}^{n}$. Let $N$ be a unit normal field on $M^{2 n-1}$ and $A=A_{N}$ the corresponding shape operator. Then $t(x)=\operatorname{rank}\left(A_{x}\right)$ is the type number of $M^{2 n-1}$ at $x \in M^{2 n-1}$. Let $\operatorname{Isom}\left(M^{2 n-1}\right)$ be the group of isometries of $M^{2 n-1}$. Then $M^{2 n-1}$ is homogeneous if for any $x, y$ $\in M^{2 n-1}$ there exists $f \in I \operatorname{Isom}\left(M^{2 n-1}\right)$ such that $f(x)=y$ and $\left(d_{x} f\right) B_{x}=B_{y}$. Examples of homogeneous real hypersurfaces of a complex Hopf manifold are furnished in section 7 . We obtain:

THEOREM 6. Let $M^{2 n-1}$ be an oriented real hypersurface of $\boldsymbol{H}_{a, c}^{n}$. If $M^{2 n-1}$ is homogeneous, then the type numbers of $M^{2 n-1}$ are either constant or $\leqq 1$ everywhere on $M^{2 n-1}$.

\section{Real hypersurfaces with two principal curvatures.}

Let $\Psi: M^{2 n-1} \rightarrow \boldsymbol{H}_{a, c}^{n}$ be an orientable real hypersurface of the $P_{0} K$-manifold $\boldsymbol{H}_{a, c}^{n}$. Let $N$ be a unit normal field on $M^{2 n-1}$. We set $A=A_{N}$ for simplicity. Let $\lambda_{j} \in C^{\infty}\left(M^{2 n-1}\right), \quad 1 \leqq j \leqq 2 n-1$, be the principal curvatures of $M^{2 n-1}$, i.e. $\operatorname{Spec}\left(A_{x}\right)=\left\{\lambda_{j}(x) / 1 \leqq j \leqq 2 n-1\right\}, x \in M^{2 n-1}$. At this point, we may prove Theorem 4. This is done in several steps, as follows. Let us assume that $A$ has exactly two distinct eigen-values at each point of $M^{2 n-1}$, say $\lambda, \mu \in C^{\infty}\left(M^{2 n-1}\right)$. Let $p, q$ be multiplicities of $\lambda, \mu$ respectively $(p+q=2 n-1)$; it is known that $p, q$ are constant. Let $T_{\lambda, x}$ be the eigen-space of $A_{x}$ corresponding to $\lambda(x)$. Then $T_{\lambda}$ (respectively $T_{\mu}$ ) is a smooth $p$-distribution (respectively $q$ distribution) on $M^{2 n-1}$, by standard arguments.

STEP 1. $T_{\lambda}, T_{\mu}$ are integrable.

Let $X, Y \in \Gamma\left(T_{\lambda}\right)$. One may use $g_{0}(h(X, Y), N)=g(A X, Y)$ such as to produce the following version of the Codazzi equation (29), that is :

$$
\left(\nabla_{X} A\right) Y-\left(\nabla_{Y} A\right) X=\frac{1}{4}\{\omega(Y) X-\omega(X) Y\} \omega_{0}(N)
$$

Using (32), as $\nabla$ is torsion-free, one obtains :

$$
(A-\lambda)[X, Y]=\omega_{\lambda}(X) Y-\omega_{\lambda}(Y) X
$$


where:

$$
\omega_{\lambda}=d \lambda+\frac{1}{4} \omega_{0}(N) \omega
$$

By the uniquences of the direct sum decomposition:

$$
T_{x}\left(M^{2 n-1}\right)=T_{\lambda, x} \oplus T_{\mu, x}
$$

for $x \in M^{2 n-1}$, it follows that both sides of (33) must vanish, i. e. $[X, Y] \in \Gamma\left(T_{\lambda}\right)$.

Remark. If $\omega_{\lambda}$ has no singular points and $B^{\perp}$ is parallel in the normal bundle then the Pfaffian equation $\omega_{\lambda}=0$ determines a codimension one foliation $\Phi_{\lambda}$ on $M^{2 n-1}$. If, moreover, the multiplicity of $\lambda$ is $p \geqq 2$ then each leaf of $T_{\lambda}$ is a submanifold of some leaf of $\mathscr{F}_{\lambda}$. Indeed, if $\nabla^{\perp} B^{\perp}=0$, then $d\left(\omega_{0}(N)\right)=0$. Consequently $\omega_{\lambda}$ is a closed 1 -form on $M^{2 n-1}$. If $p \geqq 2$ then $\omega_{\lambda}(X) Y=\omega_{\lambda}(Y) X$, for any $X, Y \in \Gamma\left(T_{\lambda}\right)$, yields $\left(\omega_{\lambda}\right)_{x}=0$ on $T_{\lambda, x}$.

STEP 2. Assume $\lambda, \mu$ are constant, $\lambda \neq \mu$. Then each leaf of $T_{\mu}$ (respectively of $T_{\lambda}$ ) is totally-umbilical, both as a submanifold of $M^{2 n-1}$ and as a submanifold of $\boldsymbol{H}_{a, c}^{n}$.

Let $X \in \Gamma\left(T_{\lambda}\right), Y \in \Gamma\left(T_{\mu}\right)$. The Codazzi equation (32) and comparison between the $T_{\mu}$-components lead to:

$$
(A-\lambda) \nabla_{Y} X+\frac{1}{4} \omega_{0}(N) \omega(X) Y=0 .
$$

By Step $1, T_{\mu}$ is integrable. Let $L$ be a leaf of $T_{\mu}$ and $i: L \rightarrow M^{2 n-1}$ the natural inclusion. Let $h_{\imath}$ be its second fundamental form. Taking the inner product of (34) with $Z \in T_{\mu}$ one obtains :

$$
(\lambda-\mu) g\left(X, \nabla_{Y} Z\right)+\frac{1}{4} \omega_{0}(N) \omega(X) r(Y, Z)=0
$$

where $\gamma=i^{*} g$. Let $\iota_{\lambda, x}: T_{x}\left(M^{2 n-1}\right) \rightarrow T_{\lambda, x}, x \in L$, be the natural projection. Then (35) leads to :

$$
(\lambda-\mu) h_{\imath}=-\frac{1}{4} \omega_{0}(N) \gamma \otimes c_{\lambda} B
$$

i.e. $L$ is totally-umbilical in $M^{n-1}$. Let $h$, be the second fundamental form of $j: L \rightarrow \boldsymbol{H}_{a, c}^{n}, j=\Psi \circ i$. The Gauss formula (26) and (36) give :

$$
h_{j}=r \otimes\left\{-\frac{1}{4(\lambda-\mu)} \omega_{0}(N) \iota_{\lambda} B+\mu N\right\}
$$

i.e. $L$ is totally-umbilical in $\boldsymbol{H}_{a, c}^{n}$.

STEP 3. $T_{\lambda}, T_{\mu}$ are parallel (with respect to $\nabla$ ) provided that $p \geqq 2$ and $q \geqq 2$. 
As $p \geqq 2$ one has $\omega_{\lambda}(X)=0$ for any $X \in \Gamma\left(T_{\lambda}\right)$. Next, by (34) and $\lambda=$ const., one obtains :

and :

$$
(A-\lambda) \nabla_{Y} X=0
$$

$$
\omega_{0}(N) \omega(X)=0
$$

for any $X \in \Gamma\left(T_{\lambda}\right), Y \in \Gamma\left(T_{\mu}\right)$. Similarly to (38), one also has $(A-\mu) \nabla_{X} Y=0$. This is enough for establishing parallelism of both $T_{\lambda}, T_{\mu}$. For instance, if $Z \in T_{\lambda}$, as $\nabla g=0$, one has $0=g\left(\nabla_{Z} X, Y\right)+g\left(X, \nabla_{Z} Y\right)$ and thus $\nabla_{z} X \in \Gamma\left(T_{\lambda}\right)$, etc.

Remark. $B^{\perp}$ has singular points. The proof is by contradiction. Suppose $B_{x}^{\perp} \neq 0$, for any $x \in M^{2 n-1}$. Then (39) yields $\omega=0$ on $T_{\lambda}$. Since, similarly to (39), one may obtain $\omega_{0}(N) \omega(Y)=0$, for any $Y \in \Gamma\left(T_{\mu}\right)$, it is clear that $\omega \equiv 0$. This has the following geometric meaning. Let $\mathscr{F}_{0}$ be the canonical foliation of $\boldsymbol{H}_{a, c}^{n}$ (see [49]) whose leaves are the maximal connected integral manifolds of the Pfaffian equation $\omega_{0}=0$. Thus $M^{2 n-1}$ appears to be a leaf of $\mathscr{I}_{0}$. Consequently $M^{2 n-1}$ would be (by (31)) totally-geodesic in $\boldsymbol{H}_{a, c}^{n}$ and therefore $\lambda=$ $\mu=0$, a contradiction.

STEP 4. The following formula holds:

$$
p q\left(\lambda \mu+\frac{c^{2}}{4}\right)=\frac{1}{4}\left\|\sqrt{q}\left(\iota_{\lambda} B\right)+\sqrt{p}\left(\iota_{\mu} B\right)\right\|^{2}
$$

Let $X \in \Gamma\left(T_{\lambda}\right), Y \in \Gamma\left(T_{\mu}\right)$. By Step 3, $T_{\mu}$ is parallel, so that $R(X, Y) Y \in \Gamma\left(T_{\mu}\right)$. Thus :

$$
g(R(X, Y) Y, X)=0
$$

On the other hand, by the Gauss equation (28), one obtains:

$$
\begin{aligned}
R(X, Y) Y= & \left(\lambda \mu+\frac{c^{2}}{4}\right)\|Y\|^{2} X \\
& +\frac{1}{4}\left\{[\omega(X) Y-\omega(Y) X] \omega(Y)-\|Y\|^{2} \omega(X) B\right\}
\end{aligned}
$$

Take the inner product of (42) with $X$ and use (41). This procedure leads to:

$$
\left(\lambda \mu+\frac{c^{2}}{4}\right)\|X\|^{2}\|Y\|^{2}-\frac{1}{4}\left\{\omega(Y)^{2}\|X\|^{2}+\omega(X)^{2}\|Y\|^{2}\right\}=0
$$

Let $\left\{E_{i}\right\}_{1 \leqslant i \leqslant 2 n-1}$ be a (locally defined) orthonormal frame on $M^{2 n-1}$, chosen in such a way that $\left\{E_{\alpha}\right\}_{1 \leqq \alpha \leqq p}$ and $\left\{E_{a+p}\right\}_{1 \leqq a \leqq q}$ are respectively frames of $T_{\lambda}, T_{\mu}$. Use (43) for $X=E_{\alpha}, Y=E_{a+p}$ and take the sum (over $\left.\alpha, a\right)$ in the resulting equation. This yields (40), Q.E.D.

At this point we may prove Theorem 4 . We distinguish two possibilities. Either $B^{\perp} \equiv 0$, i.e. $M^{2 n-1}$ is tangent to $B_{0}$, or there exists $x_{0} \in M^{2 n-1}$ such that $B_{0, x_{0}} \neq 0$. Then, by (39), $\omega_{x_{0}}=0$ on $T_{\lambda, x_{0}}$. Similarly $\omega_{x_{0}}=0$ on $T_{\mu, x_{0}}$, i.e., $\omega_{x_{0}} \equiv 0$. Thus $B_{x_{0}}=0$ and by Step 4 one has : 


$$
\lambda \mu+\frac{c^{2}}{4}=0 .
$$

By Step 3 each leaf $L$ of $T_{\mu}$ is totally geodesic in $M^{2 n-1}$. Then (36) gives $h_{\jmath}=\mu r \otimes N$. Therefore, the mean curvature vector $H_{\jmath}$ of $L \rightarrow \boldsymbol{H}_{a, c}^{n}$ is $H_{\jmath}=\mu N$, and consequently $H_{j}$ is parallel, To prove that $L$ is an extrinsic sphere of $\boldsymbol{H}_{a, c}^{n}$ we still have to show that $H_{3} \neq 0$. Suppose $H_{J}=0$. Then $\mu=0$ and, by (43), $g_{0}$ follows to be a Kaehler metric, a contradiction. Finally, we apply a result of [28], p. 182, vol. I, such as to conclude that $M^{2 n-1}$ is locally isometric to a product $L \times L^{\prime}$, where $L$ (respectively $L^{\prime}$ ) is a leaf of $T_{\lambda}$ (respectively of $T_{\mu}$ ).

Remark. By the Ricci equation (30) any leaf $L$ of $T_{\lambda}$ (being totally umbilical) has a flat normal connection as a submanifold of $\boldsymbol{H}_{a, c}^{n}$.

\section{Quasi-Einstein real hypesurfaces.}

Let $\Psi: M^{m} \rightarrow \boldsymbol{H}_{a, c}^{n}$ be an isometric immersion of a real $m$-dimensional Riemannian manifold $\left(M^{m}, g\right)$ in the $P_{0} K$-manifold $\boldsymbol{H}_{a, c}^{n}$. Let Ric be the Ricci tensor of $M^{m}$. If :

$$
R i c=\rho g-\frac{m-2}{4} \omega \otimes \omega
$$

where $\omega=\Psi^{*} \omega_{0}$ for some $\rho \in C^{\infty}\left(M^{m}\right)$, then $M^{m}$ is said to be quasi-Einstein.

Remark. See [22], p. 118. Nevertheless, the definition in [22] is more general, i.e. it is requested that $R i c=a g+b \omega \otimes \omega$, for some $a, b \in C^{\infty}\left(M^{m}\right)$.

Let $M^{2 n-1}$ be a quasi-Einstein orientable real hypersurface of $\boldsymbol{H}_{a, c}^{n}, n \geq 2$. Firstly, we need to establish the following:

LEMMA 1. If $M^{2 n-1}$ is tangent to the Lee field of $\boldsymbol{H}_{a, c}^{n}$ then $\rho$ is constant.

Proof. Set $m=2 n-1$. We follow the ideas in [28], vol. I, p. 293. Suitable contraction of indices in the second Bianchi identity (satisfied by the curvature of $M^{2 n-1}$ ) leads to :

$$
r_{1 k}-2 g^{i \jmath} R_{i k \mid \jmath}=0
$$

where $r=g^{i j} R_{\imath}$, denotes the scalar curvature, while a bar stands for covariant derivatives. Next (45) furnishes:

$$
R_{i k \mid \jmath}=\rho_{\mid j} g_{i k}-\frac{m-2}{4}\left\{\omega_{i \mid j} \omega_{k}+\omega_{k \mid j} \omega_{i}\right\}
$$

As $B^{\perp}=0$, (31) yields $\omega_{i \downarrow \jmath}=0$. Then $(46)-(47)$ show that $(m-2) \rho_{\mid k}=0$. Also $m \geqq 3$ as $n \geqq 2$. Q.E. D. 
LEMMA 2. The principal curvatures $\lambda_{\imath}$. $1 \leqq i \leqq 2 n-1$, of $M^{2 n-1}$ satisfy the quadratic equation.

$$
t^{2}-m H t+\rho-\frac{m-2}{4} c^{2}=0
$$

provided that $B^{\perp}=0$. Therefore, at any point of $M^{2 n-1}$ there are at most two distınct principal curvatures. Here $m H=$ Trace $(A)$.

By suitable contraction of indices in (28) one obtains :

$$
\begin{aligned}
\operatorname{Ric}(X, Y)= & m H g(A X, Y)-g\left(A^{2} X, Y\right) \\
& +\frac{1}{4}\left\{(m-1) c^{2}-\|\omega\|^{2}\right\} g(X, Y)-\frac{m-2}{4} \omega(X) \omega(Y)
\end{aligned}
$$

Let $\left\{E_{\imath}\right\}_{1 \leqq \imath \leqq m}$ be principal directions on $M^{2 n-1}$ corresponding to the principal curvatures $\left\{\lambda_{\imath}\right\}_{1 \leqq \imath \leqq m}$. Let us use (45), (49) for $X=Y=E_{\imath}$. This procedure gives :

$$
\rho=m H \lambda_{i}-\lambda_{i}^{2}+\frac{1}{4}\left\{(m-1) c^{2}-\|\omega\|^{2}\right\}
$$

i.e. each $\lambda_{2}$ satisfies (48), (as $B^{\perp}=0$ yields $\|\omega\|=c$ ). Q.E.D.

At this point we may prove our Theorem 5 . By Lemma 2 , let $\lambda, \mu$ be the two principal curvatures on $M^{2 n-1}$. If $\rho>(2 n-3 / 4) c^{2}$ then, by (48), $\lambda \mu>0$ and thus $\lambda, \mu$ have the same sign. Let us show that $\lambda=\mu$, i. e. $M^{2 n-1}$ is umbilical. The proof is by contradiction. Suppose $\lambda \neq \mu$. Then $\lambda+\mu=\operatorname{Trace}(A)=$ $p \lambda+(m-p) \mu$, i.e. $p=1, m-p=1$. Thus $m=2$, a contradiction ( $m$ is odd).

If $\rho=(2 n-3) c^{2} / 4$ then $\lambda \mu=0$. Then either $\lambda=\mu=0$, i. e. $M^{2 n-1}$ is totally geodesic, or $\lambda \neq 0, \quad \mu=0$. In the last case $p \lambda=\operatorname{Trace}(A)=m H=\lambda+\mu=\lambda$, i. e. $(p-1) \lambda=0$. Thus $p=1$.

If $\rho<(2 n-3) c^{2} / 4$ then $\lambda \mu<0$. Thus $(p-1) \lambda+(m-p-1) \mu=0,1<p<m-1$, and $\lambda \mu=\rho-(m-2 / 4) c^{2}$, and therefore $\lambda^{2}=-(m-p-1 / p-1)\left[\rho-(m-2 / 4) c^{2}\right]$, $\mu^{2}=-(p-1) /(m-p-1)\left[\rho-(m-2) c^{2} / 4\right]$, i. e. $\lambda, \mu$ are distinct and constant. By Theorem 4 , the proof is complete.

\section{Homogeneous real hypersurfaces.}

Let $G_{a}, 0<a<1$, be the discrete group of transformations of $W_{1}=\boldsymbol{R}^{n}-\{0\}$ generated by $x \mapsto a x, x \in W_{1}$. Then $G_{a}$ acts freely and properly discontinuously on $W_{1}$ so that the factor space $\boldsymbol{R} H_{a}^{n}=W_{1} / G_{a}$ turns to be a (compact) smooth manifold. This is the real Hopf manifold. It is a local similarity manifold (see [43]) carrying the untwisting metric $|x|^{-2} \delta_{i j} d x^{3} \otimes d x^{3}$. Let us denote by $\pi_{1}: W_{1} \rightarrow \boldsymbol{R} H_{a}^{n}$ the natural surjection.

Let $j: \boldsymbol{R}^{2 n-1} \rightarrow \boldsymbol{R}^{2 n}$ be the natural imbedding, i.e. 


$$
\left(x^{1}, \cdots, x^{2 n-1}\right) \longrightarrow\left(x^{1}, \cdots, x^{2 n-1}, 0, \cdots, 0\right)
$$

Let $\boldsymbol{H}_{a}^{n}$ be the complex Hopf manifold, cf. our section 1. Then :

$$
\boldsymbol{R} H_{a}^{2 n-1} \longrightarrow \boldsymbol{H}_{a}^{n}, \quad \pi_{1}(x) \longmapsto \pi(j(x))
$$

is an isometric immersion of $\left(\boldsymbol{R} H_{a}^{2 n-1},|x|^{-2} \delta_{i j}\right)$ into $\left(\boldsymbol{H}_{a}^{n}, g_{0}\right)$. Moreover $\boldsymbol{R} H_{a}^{2 n-1}$ is a totally umbilical real hypersurface of $\boldsymbol{H}_{a}^{n}$. Let $B_{0}$ be the Lee field of $\boldsymbol{H}_{a}^{n}$. Then $B=\tan \left(B_{0}\right)$ is precisely the characteristic field of $\boldsymbol{R} H_{a}^{2 n-1}$, cf. the termino$\operatorname{logy}$ in [43]. Let us show that $R H_{a}^{2 n-1}$ is homogeneous, in the sense of our section 4, i.e. $I \operatorname{Isom}\left(\boldsymbol{R} H_{a}^{2 n-1}\right)$ acts transitively on $\boldsymbol{R} H_{a}^{2 n-1}$ and 'preserves' the characteristic field. If $\pi_{1}(x), \pi_{1}(y) \in \boldsymbol{R} H_{a}^{2 n-1}$, then there exists $A \in O(2 n-1)$ such that $A x=y$. Let $\tilde{A}: \boldsymbol{R} H_{a}^{2 n-1} \rightarrow \boldsymbol{R} H_{a}^{2 n-1}$ be defined by $\pi_{1}(x) \rightarrow \pi_{1}(A x)$. It is easily seen that $\tilde{A} \in I \operatorname{som}\left(\boldsymbol{R} H_{a}^{2 n-1}\right)$ and $\left(d_{\pi_{1}(x)} \tilde{A}\right) B_{\pi_{1}(x)}=B_{\pi_{1}(y)}$.

Let $\Psi: S^{2 n-1} \rightarrow \boldsymbol{H}_{a}^{n}$ be the isometric immersion constructed in section 4 . If $x, y \in S^{2 n-1}$ then there exists $A \in O(2 n)$ such that $A x=y ; S^{2 n-1}$ is homogeneous as a real hypersurface of $\boldsymbol{H}_{a}^{n}$ since it is normal to the Lee field of $\boldsymbol{H}_{a}^{n}$.

Let us prove Theorem 6 . Let $M^{2 n-1}$ be an orientable homogeneous real hypersurface of $\boldsymbol{H}_{a, c}^{n}$. Let $x \in M^{2 n-1}$ be fixed. Let $S_{0}(x)$ be the real linear subspace consisting of all $X \in T_{x}\left(M^{2 n-1}\right)$ obeying the property:

$$
\begin{aligned}
R(X, Y)= & \frac{c^{2}}{4} X \wedge Y \\
& +\frac{1}{4}\left\{\omega \otimes[\omega(X) Y-\omega(Y) X]+\left[\omega(Y) X^{\natural}-\omega(X) Y^{\natural}\right] \otimes B\right\}
\end{aligned}
$$

for any $Y \in T_{x}\left(M^{2 n-1}\right)$. Here $b$ denotes lowering of indices by $g$. Let $X \Subset$ $\operatorname{Ker}\left(A_{x}\right)$. By the Gauss equation (28) it follows that $X$ verifies (50) for any $Y \in T_{x}\left(M^{2 n-1}\right)$, i.e. $\operatorname{Ker}\left(A_{x}\right) \subseteq S_{0}(x)$. Conversely, let $X \in S_{0}(x)$, be arbitrary. Suppose from now on that $\operatorname{rank}\left(A_{x}\right) \geqq 2$. Now $t(x)=\operatorname{dim}_{R}\left(\operatorname{Im}\left(A_{x}\right)\right)$; consequently, there exists $Y \in T_{x}\left(M^{2 n-1}\right)$ such that $A_{x} Y \neq 0$ and $\left(A_{x} X, A_{x} Y\right)=0$, where $g_{x}=$ $(\cdot, \cdot)$. As $X \in S_{0}(x)$, by (28), one obtains $A_{x} X \wedge A_{x} Y=0$ and then:

$$
\left\|A_{x} X\right\|^{2} A_{x} Y=0
$$

i. e. $X \in \operatorname{Ker}\left(A_{x}\right)$. We conclude that:

$$
S_{0}(x)=\operatorname{Ker}\left(A_{x}\right)
$$

provided that $t(x) \geq 2$. Let $y \in M^{2 n-1}, y \neq x . \quad$ As $M^{2 n-1}$ is homogeneous, let $f \in$ $\operatorname{Isom}\left(M^{2 n-1}\right)$ such that $f(x)=y,\left(d_{x} f\right) B_{x}=B_{y}$. Then $(d f) S_{0}(x) \cong S_{0}(y)$. Consequently $\operatorname{dim}_{R} S_{0}(x) \leqq \operatorname{dim}_{R} S_{0}(y)$. The reversed inequality may be proved in a similar way. Thus $t(x)=t(y)$. So, if $t(x) \geqq 2$ then $t(x)=$ const. If, in turn, $t(x)$ $\leqq 1$, for some $x \in M^{2 n-1}$, then by (28) one obtains $\operatorname{dim}_{R} S_{0}(x)=2 n-1$. Thus, by the homogeneity assumption, $\operatorname{dim}_{R} S_{0}(y)=2 n-1$, for all $y \in M^{2 n-1}$. Using again the Gauss equation it follows that $\operatorname{rank}(A) \leqq 1$ everywhere on $M^{2 n-1}, \mathrm{Q}$. E. D. 


\section{Strongly invariant and totally umbilical submanifolds.}

Let $M$ be a submanifold of the Riemannian manifold $(\bar{M}, \bar{g})$. Let $\bar{R}$ be the curvature 2 -form of $\bar{M}$. Then $M$ is an invariant submanifold (cf. K. Ogiue, [40], p. 389) if for any $x \in M$, and any $u, v \in T_{x}(M)$ the tangent space $T_{x}(M)$ is invariant under the curvature transformation $\bar{R}_{x}(u, v): T_{x}(\bar{M}) \rightarrow T_{x}(\bar{M})$, that is $\bar{R}_{x}(u, v)\left(T_{x}(M)\right) \subset T_{x}(M)$. Also $M$ is termed strongly invariant if $T_{x}(M)$ is invariant under $\bar{R}_{x}(\bar{u}, \bar{v})$, for any $\bar{u}, \bar{v} \in T_{x}(\bar{M}), x \in M$. For instance, any submanifold of a real space form is invariant, see $[40]$, p. 390 . Also each extrinsic sphere (i.e. totally umbilical submanifold whose non zero mean curvature vector is parallel in the normal bundle), is invariant, by the Codazzi equation, see e.g. B. Y. Chen [5], p. 329. We may finally mention that since any invariant submanifold of a complex space form is either holomorphic or totally real, cf. [7], p. 260, the totally umbilical submanifolds of complex space forms have been completely classified, see Theorem 1 in B. Y. Chen \& K. Ogiue, [8], p. 225 .

The present section aims to the study of (strongly) invariant submanifolds of complex Hopf manifolds (with the Boothby metric). We may formulate the following.

THEOREM 7. Let $M^{m}$ be a real $m$-dimensional, $1<m<2 n$, strongly invariant submanifold of the complex Hopf manifold $\boldsymbol{H}_{a}^{n}, n>1$. Then either $M^{m}$ is normal to the Lee field of $\boldsymbol{H}_{a}^{n}$ or $M^{m}$ is a real hypersurface of $\boldsymbol{H}_{a}^{n}$.

THEOREM 8. Let $M^{m}$ be a totally umbilical submanifold of the complex Hopf manifold $\boldsymbol{H}_{a}^{n}$. If $M^{m}$ is tangent to the Lee field of $\boldsymbol{H}_{a}^{n}$ then $M^{m}$ is either totally geodesic or an extrinsic sphere.

Let $M^{m}$ be a submanifold of $\boldsymbol{H}_{a}^{n}$; the Codazzi equation of $M^{m}$ in $\boldsymbol{H}_{a}^{n}$ may be written as follows:

$$
\operatorname{nor}(\bar{R}(X, Y) Z)=\frac{1}{4}\{g(X, Z) \omega(Y)-g(Y, Z) \omega(X)\} B^{\perp}
$$

Therefore, any submanifold of $\boldsymbol{H}_{a}^{n}$ which is tangent to the Lee field is invariant. Let $\Phi_{0}$ be the canonical foliation of $\boldsymbol{H}_{a}^{n}$, see section 5 . By (52), any leaf of $\mathscr{F}_{0}$ is an invariant submanifold of $\boldsymbol{H}_{a}^{n}$. Conversely, one obtains :

Proposition. Let $M^{m}, m \geqq 2$, be an invariant submanifold of $\boldsymbol{H}_{a}^{n}$, Then either $M^{m}$ is tangent to the field of $\boldsymbol{H}_{a}^{n}$, or, if $B_{x}^{\perp} \neq 0$ at some point $x \in M^{m}$, then $x$ is a singular point of the induced 1-form $\omega$ on $M^{m}$.

Proof. If $M^{m}$ is invariant, then by (52) one has:

$$
\left\{(u, w) \omega_{x}(v)-(v, w) \omega_{x}(u)\right\} B_{x}^{\frac{1}{x}}=0
$$


for any $u, v, w \in T_{x}\left(M^{m}\right)$ and any $x \in M^{m}$. Here $g_{x}=(\cdot, \cdot)$. We distinguish two cases: either $B_{x}^{1}=0$ for all $x \in M^{m}$, i. e. $M^{m}$ is tangent to $B_{0}$, or there exists $x_{0} \in M^{m}$ such that $B_{x_{0}}^{1} \neq 0$. If this is the case, let $u \in T_{x_{0}}\left(M^{m}\right)$ be arbitrary. Since $m \geqq 2$ we may consider $v=w,\|v\|=1,(u, v)=0, v \in T_{x_{0}}\left(M^{m}\right)$. By (53) one obtains $\omega_{x_{0}}(u)=0$, for all $u$, Q. E. D.

We proceed by proving our Theorem 7. Suppose $M^{m}$ is strongly invariant. Then for any tangent vector fields $\bar{X}, \bar{Y}$ on $\boldsymbol{H}_{a}^{n}$, respectively any tangent vector field $Z$ on $M^{m}$, one has :

$$
\begin{aligned}
& \left\{g_{0}(\bar{Y}, Z)-\frac{1}{4} \omega_{0}(\bar{Y}) \omega(Z)\right\} \operatorname{nor}(\bar{X}) \\
& \quad-\left\{g_{0}(\bar{X}, Z)-\frac{1}{4} \omega_{0}(\bar{X}) \omega(Y)\right\} \operatorname{nor}(\bar{Y}) \\
& \quad+\frac{1}{4}\left\{g_{0}(\bar{X}, Z) \omega_{0}(\bar{Y})-g_{0}(\bar{Y}, Z) \omega_{0}(\bar{X})\right\} B^{\perp}=0
\end{aligned}
$$

as a consequence of (16), (where $c=2$ ). K. Ogiue, [40], p. 393, has shown that there exist no strongly invariant submanifolds in a Riemannian manifold of non zero constant curvature. Following the scheme of proof of Theorem 3.4, in [40], p. 393, let $\bar{X}=X=Z, \bar{Y}=\xi$, where $X$ is tangential, while $\xi$ is normal. Then (54) leads to :

$$
\left\{\frac{1}{4} \omega(X)^{2}-\|X\|^{2}\right\} \xi+\frac{1}{4}\|X\|^{2} \omega_{0}(\xi) B^{\perp}=0
$$

We distinguish two possibilities. Either $\omega \equiv 0$, i.e. $M^{m}$ is normal to $B_{0}$, or there exists $x_{0} \in M^{m}$ such that $\omega_{x_{0}} \neq 0$. If this is the case then $B_{x_{0}} \neq 0$ and, since $m \geqq 2$, we may choose $u \in T_{x_{0}}\left(M^{m}\right),\|u\|=1,\left(u, B_{x_{0}}\right)=0$. Apply (55) for $X_{x_{0}}=u$. If follows that:

$$
\omega_{0, x_{0}}(z) B_{x_{0}}^{\perp}=4 z
$$

for any $z \in E(\Psi)_{x_{0}}$. Clearly $B_{x_{0}}^{\perp} \neq 0$; otherwise, by (56) one would have $m=2 n$, a contradiction. Thus, by $(56), E(\Psi)_{x_{0}}$ is spanned by $B_{x_{0}}^{\perp}$, i.e. $\operatorname{codim}\left(M^{m}\right)=1$. Q.E.D.

Let $M^{m}$ be a totally umbilical submanifold of $\boldsymbol{H}_{a}^{n}$. By the Codazzi equation (e.g. (2.7) in [4], p. 46) and umbilicity :

$$
\operatorname{nor}(\bar{R}(X, Y) Z)=g(Y, Z) \nabla_{X}^{\perp} H-g(X, Z) \nabla_{\bar{Y}}^{\frac{1}{X}} H
$$

As $m \geqq 2$, for fixed $X$ let us choose $Y$ such that $\|Y\|=1, g(X, Y)=0$; then (57) yields $\operatorname{nor}(\bar{R}(X, Y) Y)=\nabla_{\bar{X}}^{\frac{1}{H}} H$. This and (52) yield:

$$
\nabla_{\bar{X}}^{\perp} H=-\frac{1}{4} \omega(X) B^{\perp}
$$

The situations in Theorem 8 correspond to $H=0, H \neq 0$, respectively. 
Remarks. 1) Let $M^{2 m}$ be an even dimensional extrinsic sphere of the 1.c.K. manifold $M^{2 n}$, having a flat normal connection. If $\omega_{0}=0$, (i.e. the ambient space is Kaehler) then the simply-connected complete extrinsic spheres with a flat normal connection have been classified by B. Y. Chen, [5]. Actually, any such $M^{2 m}$ is isometric to the sphere $S^{2 m}(1 / k)$ of radius $1 / k, k=\|H\|=$ const. See Theorem 1 in [5], p. 328. The classification problem for the case of a 1.c.K. ambient space is not as yet solved.

2) The key ingredient in the proof of Theorem 1 of [5], p. 328, is a result of M. Obata, [39], p. 334, asserting that a complete Riemannian manifold $M^{p}$ of dimension $p \geqq 2$ is isometric to the sphere $S^{p}(1 / r)$ if and only if the differential equation $\left(\nabla_{X} d f\right) Y=-r^{2} g(X, Y) f$ admits some non constant solution $f \in C^{\infty}\left(M^{p}\right)$. Let $M^{2 m}$ be a simply-connected extrinsic sphere in the 1.c. K. manifold $M^{2 n}$, having a flat normal connection. While leaving the classification problem open, one may show that the differential equation:

$$
\left(\nabla_{X} d f\right) Y+\frac{1}{2} X(f) \omega(Y)=-k^{2} g(X, Y) f
$$

admits non constant solutions $f \in C^{\infty}\left(M^{2 m}\right)$, provided that $M^{2 m}$ is tangent both to the Lee and anti-Lee (i. e. $A_{0}=-J B_{0}$ ) vector fields of $M^{2 n}$.

3) It is expected that the existence of solutions of (59) may be exploited on the line in [39]. While this is not as yet available, let us prove the result stated in the previous remark. Since $M^{2 m}$ is taken simply-connected, the assumption $R^{\perp}=0$ is equivalent to the existence of a frame in the normal bundle consisting of mutually orthogonal parallel unit vector fields. Let $N=k^{-1} H$. Then $N$ is a parallel unit normal field. Choose a frame $\left\{N_{a}\right\}_{1 \leqq a \leqq 2 a-2 m}$ in $E(\Psi)$ such that $N_{1}=N, g_{0}\left(N_{a}, N_{b}\right)=\delta_{a b}, \nabla^{\perp} N_{a}=0$, for all $1 \leqq a, b \leqq 2 n-2 m$. Next, let us construct the functions $f_{a} \in C^{\infty}\left(M^{2 m}\right)$ by setting $f_{a}=g_{0}\left(J N, N_{a}\right), 2 \leqq a \leqq$ $2 n-2 m$. By (11) and (15) one has:

$$
\bar{\nabla}_{X} J \xi=J \bar{\nabla}_{X} \xi+\frac{1}{2}\left\{\theta_{0}(\xi) X-\omega_{0}(\xi) J X-\Omega_{0}(X, \xi) B_{0}\right\}
$$

for any tangential vector field $X$, respectively any normal section $\xi$ in $E(\Psi)$. Here $\theta_{0}=\omega_{0} \circ \mathrm{J}$ is the anti-Lee form. Set $A^{\perp}=$ nor $\left(A_{0}\right)$. We establish the following :

Lemma 1. If $A^{\perp}=0, B^{\perp}=0$ then for each tangent vector field $X$ on $M^{2 m}$ one has:

$$
X\left(f_{a}\right)=k \Omega_{0}\left(X, N_{a}\right)
$$

for any $a \geqq 2$.

Proof. Using (60) we have 


$$
\begin{aligned}
X\left(f_{a}\right) & =X\left(g_{0}\left(J N, N_{a}\right)\right) \\
& =g_{0}\left(\bar{\nabla}_{X} J N, N_{a}\right)+g_{0}\left(J N, \bar{\nabla}_{X} N_{a}\right)=g_{0}\left(J \bar{\nabla}_{X} N, N_{a}\right) \\
& +\frac{1}{2}\left\{\omega_{0}(J N) g_{0}\left(X, N_{a}\right)-\omega_{0}(N) g_{0}\left(J X, N_{a}\right)-g_{0}(X, J N) \omega_{0}\left(N_{a}\right)\right\} .
\end{aligned}
$$

Use was made of :

$$
\bar{\nabla}_{X} N_{a}=-A_{N_{a}}+\nabla_{\bar{X}}^{\perp} N_{a}=-g_{0}\left(N_{a}, H\right) X=0
$$

for any $a \geqq 2$, (as a consequence of (27) and umbilicity). Thus :

$$
X\left(f_{a}\right)=k g_{0}\left(X, J N_{a}\right)+\frac{1}{2}\left\{\omega_{0}(N) \Omega_{0}\left(X, N_{a}\right)-\omega_{0}\left(N_{a}\right) \Omega_{0}(X, N)\right\}
$$

for $a \geqq 2$. As $M^{2 m}$ is tangent to both the Lee and anti-Lee vector fields, the Lee and anti-Lee forms vanish on normal vectors. Thus (62) leads to (61).

LEMMA 2. There exists $2 \leqq a \leqq 2 n-2 m$ such that at least one function $f_{a}$ is not constant.

Proof. Suppose the functions $f_{a}, a \geqq 2$, are simultaneously constant. Then, by our Lemma 1 , it follows $0=X\left(f_{a}\right)=k g\left(X, J N_{a}\right)$, i. e. $J N_{a}$ are still normal, $a \geqq 2$. Consequently, the linear space $W_{x}$ spanned by $N_{a, x}, J_{x} N_{a, x}, a \geqq 2$, over the reals, is a $J_{x}$-invariant subspace of $E(\Psi)_{x}$. As $W_{x}$ carries the complex structure $J_{x}$, its algebraic dimension has to be even, i. e. $\operatorname{dim}_{R} W_{x}>2 n-2 m-1$. Thus $W_{x}=E(\Psi)_{x}, x \in M^{2 m}$, i.e. $M^{2 m}$ follows to be a holomorphic submanifold of the 1.c.K. manifold $M^{2 n}$. Thus $H=-(1 / 2) B^{\perp}$, i.e. $M^{2 m}$ is minimal, a contradiction.

Finally, let us show that each $f_{a}, a \geqq 2$, is a solution of (59). By Lemma 1 and (26) one has :

$$
\begin{aligned}
X\left(Y\left(f_{a}\right)\right)= & k X\left(Y_{0}\left(J N_{a}\right)\right)=k g_{0}\left(\bar{\nabla}_{X} Y, J N_{a}\right)+k g_{0}\left(Y, \bar{\nabla}_{X} J N_{a}\right) \\
= & k g_{0}\left(\nabla_{X} Y, J N_{a}\right)+k g_{0}\left(h(X, Y), J N_{a}\right)+k g_{0}\left(Y, J \bar{\nabla}_{X} N_{a}\right) \\
& +\frac{k}{2}\left\{\omega_{0}\left(J N_{a}\right) g_{0}(X, Y)-g_{0}\left(X, J N_{a}\right) \omega(Y)\right\}
\end{aligned}
$$

or (by (61) and umbilicity):

$$
\begin{aligned}
X\left(Y\left(f_{a}\right)\right)= & \left(\nabla_{X} Y\right)\left(f_{a}\right)-k^{2} g(X, Y) f_{a} \\
& +\frac{k}{2}\left\{g(X, Y) \theta_{0}\left(N_{a}\right)-\Omega_{0}\left(X, N_{a}\right) \omega(Y)\right\}
\end{aligned}
$$

Now (63) shows that $f_{a}$ is a solution of (59), provided that $A^{\perp}=0$, Q. E. D. 


\section{REFERENCES}

[1] W. Boothby, Some fundamental formulas for Hermitian manifolds with nonvanishing torsion, Am. J. Math., 76 (1954), 509-534.

[2] E. BRIESKORN, Beispiele zur Differenzialtopologie von Singularitaten, Inventiones Math., 2 (1966), 1-14.

[3] E. BRieskorn and A. VAN De Ven, Some complex structures on products of homotopy spheres, Topology, 7 (1968), 389-393,

[4] B. Y. Chen, Geometry of submanifolds, Pure and Appl. Math., M. Dekker, Inc., New York, 1973, 298 p.

[5] B. Y. CHEN, Extrinsic spheres in Kaehler manifolds. I-II., Michigan Math. J., 24 (1977), 97-102.

[6] B. Y. CHEN, Extrinsic spheres in compact symmetric spaces are intrinsic spheres, Michigan Math. J., 24 (1977), 265-271.

[7] B. Y. Chen And K. Ogiue, On totally-real submanifolds, Trans. A. M.S., 193 (1974), 257-266.

[8] B. Y. Chen and K. Ogiue, Two theorems on Kaehler manifolds, Michigan Math. J., 21 (1974), 225-229.

[9] B. Y. CHEN AND P. PICcinn, The canonical foliations of a locally conformal Kaehler manifold, Ann. di Mat. pura appl., 141 (1985), 283-305,

[10] J. L. CABRERIzo AND M.F. ANDRES, $C R$ submanifolds of a locally conformal Kaehler manifold, in Diff. Geometry (L. A. Cordero, ed.), Research Notes in Math., 131 (1985), 17-33, Pittman Adv. Publ. progr., Boston-London-Melbourne.

[11] A. Dold, Lectures on algebraic topology, Springer-Verlag, 1980, Berlin-Heidelberg-New York.

[12] S. Dragomir, On submanifolds of Hopf manifolds, Israel J. Math., (2) 61 (1988), 98-110.

[13] S. DragomiR, Cauchy-Riemann submanifolds of locally conformal Kaehler manifolds, I-II, Geometriae Dedicata, 28 (1988), 181-197, Atti Sem. Mat. Fis. Univ. Modena, 37 (1989), 1-11.

[14] S. DRAGomiR, Totally-real submanifolds of generalized Hopf manifolds, Le Matematiche, XLII (1987), 3-10.

[15] S. DRAGomir and R. Grimaldi, Isometric immersions of Riemann spaces in a real Hopf manifold, Journ. de Mathem. pures appl., 68 (1989), 355-364.

[16] R.H. Escobales, Riemannian submersions with totally geodesic fibres, J. Diff. Geom., 10 (1975), 253-276.

[17] R.H. Escobales, Riemannian submersions from complex projective space, J. Diff. Geom., 13 (1978), 93-107.

[18] D. Ferus, Totally geodesic foliations, Math. Ann., 188 (1970), 313-316.

[19] G. Gigante, Symmetries on compact pseudohermitian manifolds, Rend. Circolo Matem. Palermo, 36 (1987), 148-157.

[20] S. Goldberg, Curvature and homology, Pure and Appl. Math., Academic Press, 1962, New York-London.

[21] S. Goldberg, On the topology of compact contact manifolds, Tohoku Math. J., 20 (1968), 106-110.

[22] S. Goldberg and I. Vaisman, On compact locally conformal Kaehler manifolds with non-negative sectional curvature, Ann. Fac. Sci. Toulouse, 2 (1980), 117123. 
[23] R. HERMANN, A sufficient condition that a mapping of Riemannian manifolds be a fibre bundle, Proc. A. M.S., 11 (1960), 236-243.

[24] S. IAnus, K. Matsumoto and L. ORneA, Complex hypersurfaces of a generalized Hopf manifold, Publication de l'Inst. Math., N. S., 42 (1987), 123-129.

[25] M. InOUE, On surfaces of classes VII , Inventiones Math., 24 (1974), 269-310.

[26] T. Kashiwada AND S. SATo, On harmonic forms in compact locally conformal Kaehler manifolds with parallel Lee form, Ann. Fac. Sci. Kinshasa, Zaire, (1) 6 (1980), 17-29.

[27] T. KASHIWADA, Some properties of locally conformal Kaehler manifolds, Hokkaido Math. J., 8 (1979), 191-198.

[28] S. Koвayashi AND K. Nomizu, Foundations of differential geometry, Interscience Publ., New York, vol. I-II, 1963, 1969.

[29] Y. Matsushima, Vector bundle valued harmonic forms and immersions of Riemannian manifolds, Osaka J. Math., 8 (1971), 1-13.

[30] K. Matsumoto, On submanifolds of locally conformal Kaehler manifolds, Bull. Yamagata Univ., (1) 11 (1984), 33-38.

[31] K. Matsumoto, On $C R$ submanifolds of locally conformal Kaehler manifolds, J. Korean Math. Soc., (1) 21 (1984), 49-61.

[32] K. Matsumoto, On locally conformal Kaehler space forms, Internat. J. Math. \& Math. Sci., (1) 8 (1985), 69-74.

[33] K. Matsumoto, On $C R$ submanifolds of locally conformal Kaehler manifolds. II., Tensor, N.S., 45 (1987), 144-150.

[34] J. W. Milnor and J.D. Stasheff, Characteristic classes, Ann. of Math. Stud., Princeton Univ. Press, No. 76, 1974, New Jersey.

[35] J. R. Munkres, Elements of algebraic topology, Addison-Wesley Publ. Co., Inc., 1984, Massachusetts.

[36] M. Namba, Automorphism groups of Hopf surfaces, Tohoku Math. J., 26 (1974), 133-157.

[37] K. Nomizu, On the spaces of generalized curvature tensor fields and second fundamental forms, Osaka J. Math., 8 (1971), 21-28.

[38] B. O'Neill, The fundamental equations of a submersion, Michigan Math. J., (4) 13 (1966), 459-469.

[39] M. OватA, Certain conditions for a Riemannian manifold to be isometric to a sphere, J. Math. Soc. Japan, 14 (1962), 333-340.

[40] K. Ogiue, On invariant immersions, Ann. Matem. pura appl., LXXX (1968), 387-397.

[41] L. ORnEA, On $C R$ submanifolds of locally conformal Kaehler manifolds, Demonstratio Math., (4) 29 (1986), 863-869.

[42] M. OKumura, Contact hypersurfaces in certain Kaehlerian manifolds, Tohoku Math. J., (1) 18 (1966), 74-102.

[43] C. Reischer and I. Vaisman, Local similarity manifolds, Ann. Matem. pura appl., 35 (1983), 279-292.

[44] P. J. RYAN, Homogeneity and some curvature conditions for hypersurfaces, Tohoku Math. J., 21 (1969), 363-388.

[45] S. SASAKI AND C.J. HsU, On a property of Brieskorn manifolds, Tohoku Math. J., 28 (1976), 67-78.

[46] Y. TAshiRo, On contact structures of hypersurfaces in almost complex manifolds. I-II., Tohoku Math. J., 15 (1963), 62-78, 167-175. 
[47] F. Tricerri, Some examples of locally conformal Kaehler manifolds, Rend. Sem. Mat. Univers. Politecn. Torino, (1) 40 (1982), 81-92.

[48] H. TsukADA, Hopf manifolds and spectral geometry, Trans. A.M.S., (2) 270 (1982), 609-621.

[49] I. VAISMAN, Locally conformal Kaehler manifolds with parallel Lee form, Rendiconti di Matem., 12 (1979), 263-284.

[50] I. Vaisman, Generalized Hopf manifolds, Geometriae Dedicata, 13 (1982), 231-255.

[51] I. VAIsman, A theorem on compact locally conformal Kaehler manifolds, Proc. A. M. S., (2) 75 (1979), 279-283.

[52] I. VAISMAN, Some curvature properties of locally conformal Kaehler manifolds, Trans. A. M. S., (2) 259 (1980), 439-447.

[53] I. Vaisman, Some curvature properties of complex surfaces, Ann. Matem. pura appl., XXXII (1982), 1-18.

[54] I. VAISMAN, On locally and globally conformal Kaehler manifolds, Trans. A.M.S., (2) 262 (1980), 533-542.

[55] B. Watson, The first Betti numbers of certain locally trivial fibre spaces. Bull. A. M.S., (3) 78 (1972), 392-393.

[56] K. Yana AND M. Kon, $C R$ submanifolds of Kaehlerian and Sasakian manifolds, Progress in Math., vol. 30, Birkhauser, 1983, Boston-Basel-Stuttgart.

State University of New York at Stony Brook

Department of Mathematics

STONY BROOK, N. Y. 11794, U.S. A. 